\title{
Recognizing Guillain-Barré Syndrome in the Primary Care Setting
}

Kristi McClellan Mantay

Eastern Virginia Medical Schoo

Elin Armeau

Eastern Virginia Medical School

Thomas Parish

Eastern Virginia Medical School

Follow this and additional works at: https://nsuworks.nova.edu/ijahsp

Part of the Medicine and Health Sciences Commons

\section{Recommended Citation}

Mantay KM, Armeau E, Parish T. Recognizing Guillain-Barré Syndrome in the Primary Care Setting. The Internet Journal of Allied Health Sciences and Practice. 2007 Jan 01;5(1), Article 5.

This Manuscript is brought to you for free and open access by the College of Health Care Sciences at NSUWorks. It has been accepted for inclusion in Internet Journal of Allied Health Sciences and Practice by an authorized editor of NSUWorks. For more information, please contact nsuworks@nova.edu. 


\title{
Recognizing Guillain-Barré Syndrome in the Primary Care Setting
}

\begin{abstract}
Purpose: Guillain-Barré Syndrome (GBS) is the most common cause of acute flaccid paralysis in adults and children in the United States. The purpose of this article is to educate Primary Care Practitioners (PCP) about GBS and the importance of its recognition in the acute care setting. Method: A review of literature examined epidemiology, pathophysiology, clinical features, and antecedent events related to primary care. Results: Studies show correlations between GBS and preceding viral-bacterial infections, and certain vaccines. However, a direct causation has not been proven. Antecedent events encountered in primary care include, but are not limited to, gastroenteritis, upper respiratory infection, cytomegalovirus, Epstein-Barr virus, and vaccinations against influenza, meningitis, and tetanus toxoid.Conclusion: PCPs should be able to recognize GBS and its triggers should a case present to their clinics. It is hoped that GBS mortality and sequela would be decreased if PCPs are more knowledgeable about this condition.
\end{abstract}




\title{
IJAHISP \\ The Internet Jourfal of Allied Health Sciences and Practice \\ http://ijahsp.nova.edu
}

A Peer Reviewed Publication of the College of Allied Health \& Nursing at Nova Southeastern University

Dedicated to allied health professional practice and education

http://ijahsp.nova.edu Vol.5 No. 1 ISSN 1540-580X

Recognizing Guillain-Barré Syndrome in the Primary Care Setting

\author{
Kristi McClellan Mantay, PA-S \\ Elin Armeau, PhD, PA-C \\ Thomas Parish, DHSc, PA-C \\ United States
}

\section{Citation:}

McClellan, K., Armeau, E., Parish, T. Recognizing Guillain-Barré Syndrome in the Primary Care Setting. The Internet Journal of Allied Health Sciences and Practice. Jan 2007, Volume 5 Number 1.

\begin{abstract}
Purpose: Guillain-Barre' Syndrome (GBS) is the most common cause of acute flaccid paralysis in adults and children in the United States. The purpose of this article is to educate Primary Care Practitioners (PCP) about GBS and the importance of its recognition in the acute care setting. Method: A review of literature examined epidemiology, pathophysiology, clinical features, and antecedent events related to primary care. Results: Studies show correlations between GBS and preceding viral-bacterial infections, and certain vaccines. However, a direct causation has not been proven. Antecedent events encountered in primary care include, but are not limited to, gastroenteritis, upper respiratory infection, cytomegalovirus, Epstein-Barr virus, and vaccinations against influenza, meningitis, and tetanus toxoid. Conclusion: PCPs should be able to recognize GBS and its triggers should a case present to their clinics. It is hoped that GBS mortality and sequela would be decreased if PCPs are more knowledgeable about this condition.
\end{abstract}

\section{Introduction}

Guillain-Barre' Syndrome (GBS), although not common, is the most common cause of acute flaccid paralysis. ${ }^{1}$ Annual incidence of GBS is approximately 1-3 cases per 100,000 persons in Europe, US, and Australia. ${ }^{2}$ GBS is an autoimmune disorder of the peripheral nervous system (PNS) with a range of presentations from mild to lifethreatening paralysis. GBS is rare; therefore many practitioners have not encountered this syndrome, are unaware of the signs and symptoms, and need further education about GBS. The etiology of GBS is unknown, yet several studies link common exposures as precipitating factors, many of which are commonly seen in the primary care setting. Vaccinations, viral infections, and certain strains of food poisoning are examples of antecedent factors of GBS. Due to severity of complications, practitioners need to be aware of what can trigger GBS, who is at risk, how to recognize early signs/symptoms, what possible prevention exists, and how to educate patients.
Symptoms are preceded by an inciting event in two-thirds of all GBS diagnoses. Respiratory infection occurred in $40 \%$ of cases prior to onset of GBS and in $20 \%$ of cases, gastroenteritis was the precipitating factor. ${ }^{2}$ Vaccinations against flu, rabies, and meningitis are also documented precipitating factors that one might see in primary care. The antecedent events are all common reasons for an office visit to the Primary Care Practitioner (PCP). GBS should be in the differential diagnosis for patients presenting with potential complications from these common preceding infections or events. This article will address antecedent events and clinical features for GBS in an effort to increase awareness of the importance in efficient diagnosis and potential complications. PCPs need to recognize GBS and its triggers because they are the gatekeepers to healthcare. Recognition of GBS by the PCP may decrease the incidence of mortality and sequela.

\section{Purpose}

To educate PCPs about Guillain-Barre' Syndrome, increase awareness of GBS, and to discuss the role that 
PCPs play in the diagnosis and treatment of GBS.

\section{Review of Literature}

The review of literature examines events that are postulated to trigger GBS and events that are known to trigger GBS. The information will be compiled to educate practitioners about GBS. The review of literature will also address background information such as epidemiology, pathophysiology, clinical features, antecedent events and their relationship to primary care.

Many practitioners may remember a lecture in medical school on GBS or acute peripheral neuropathy as their only experience with GBS. But, because GBS is a rapidly ascending peripheral neuropathy that can have potentially fatal complications, the practitioner needs to know how they (practitioner) could play an important role in early diagnosis.

\section{Epidemiology}

The incidence of GBS in the United States is $1-8 / 100,000$ persons annually. ${ }^{3}$ GBS occurs in all age groups with a slight increase in the young adult and elderly populations. ${ }^{2}$ Joseph and Tsao, ${ }^{4}$ report the youngest recorded patient as four years old and the oldest patient as 95 years old. GBS affects both males and females, but males are about 1.5 times more likely to be affected than females. ${ }^{5}$ GBS is found to be slightly more common in Caucasians than African-Americans in the United States. ${ }^{1}$

Immunocompromised patients are also at a high risk for most of the antecedent illnesses, therefore increasing risk of GBS.

According to Seneviratne, ${ }^{2}$ a cohort study suggests that the risk for GBS is increased in the first few months after giving birth, while the risk is lower during the actual pregnancy. No direct genetic link has been determined. GBS is presumed to be the person's idiosyncratic immune response to the preceding infection, in which there may be an underlying genetic basis. There has been no link to geographic location except for sporadic clusters due to outbreaks of communicable illnesses or exposure to contamination.

\section{Pathophysiology}

While the exact cause is not known, GBS is thought to be an autoimmune disease process in both the demyelinating and axonal forms of the disease. The discovery of antiganglioside antibodies is due to the relationship of infection and autoimmune properties. ${ }^{6}$ The presence of antibodies and activated T-cells that react against the peripheral myelin gives rise to the theory that GBS is a type of autoimmune pathology. Macrophages invade the Schwann cell, strip myelin from the axon, and sometimes degeneration of the axon occurs, which may be secondary to an autoimmune attack on the axon or myelin. Autoreactive T-cells are thought to be "activated" and mediate this autoimmune response by initiating the cascade to inflammation. ${ }^{7}$ Winer, proposes a possible mechanism of disease where the antiganglioside antibodies appear to cross-react with particular antigens found in the lipopolysaccharide of certain infective agents known to be GBS triggers. ${ }^{6}$ Current literature suggests that molecular mimicry is a likely mechanism of the autoimmune reaction. Molecular mimicry is when the immune system responds to specific antigens (thought to be present due to the antecedent infection) or the actual organism implicated in the preceding infection, and causes the immune system to attack similar epitopes in the peripheral nervous system. There are five distinctive subtypes of GBS that are classified according to pathology and electrodiagnostic studies (see Table 1 for subtypes and descriptions).

\section{Clinical Features}

GBS occurs in relatively healthy persons that experience an antecedent infection/event anywhere from days to weeks prior to onset of GBS symptoms. The most common infections reported in GBS cases are bacterial gastroenteritis and upper respiratory infection that was present approximately one to three weeks before the onset of any neurological symptoms. Approximately $20 \%$ of GBS patients experience a form of gastroenteritis prior to GBS diagnosis (Seneviratne, 2000). There is speculation that specific vaccinations can trigger GBS.

Persons with GBS experience a gradual symmetrical onset of paresthesia and numbness that begins at the feet and ascends at a variable rate. Progression may also include the hands and feet ascending distally to proximally, eventually leading to motor weakness then paralysis.

Sensory or autonomic dysfunction is present in about two thirds of cases, but is not necessarily present in all cases. ${ }^{8}$ Autonomic disruption can manifest in many variations such as hyper/hypotension, cardiac arrhythmias with wide variations, and other various symptoms (see Table 2). 
Table 1. Subtypes of Guillain-Barre' Syndrome

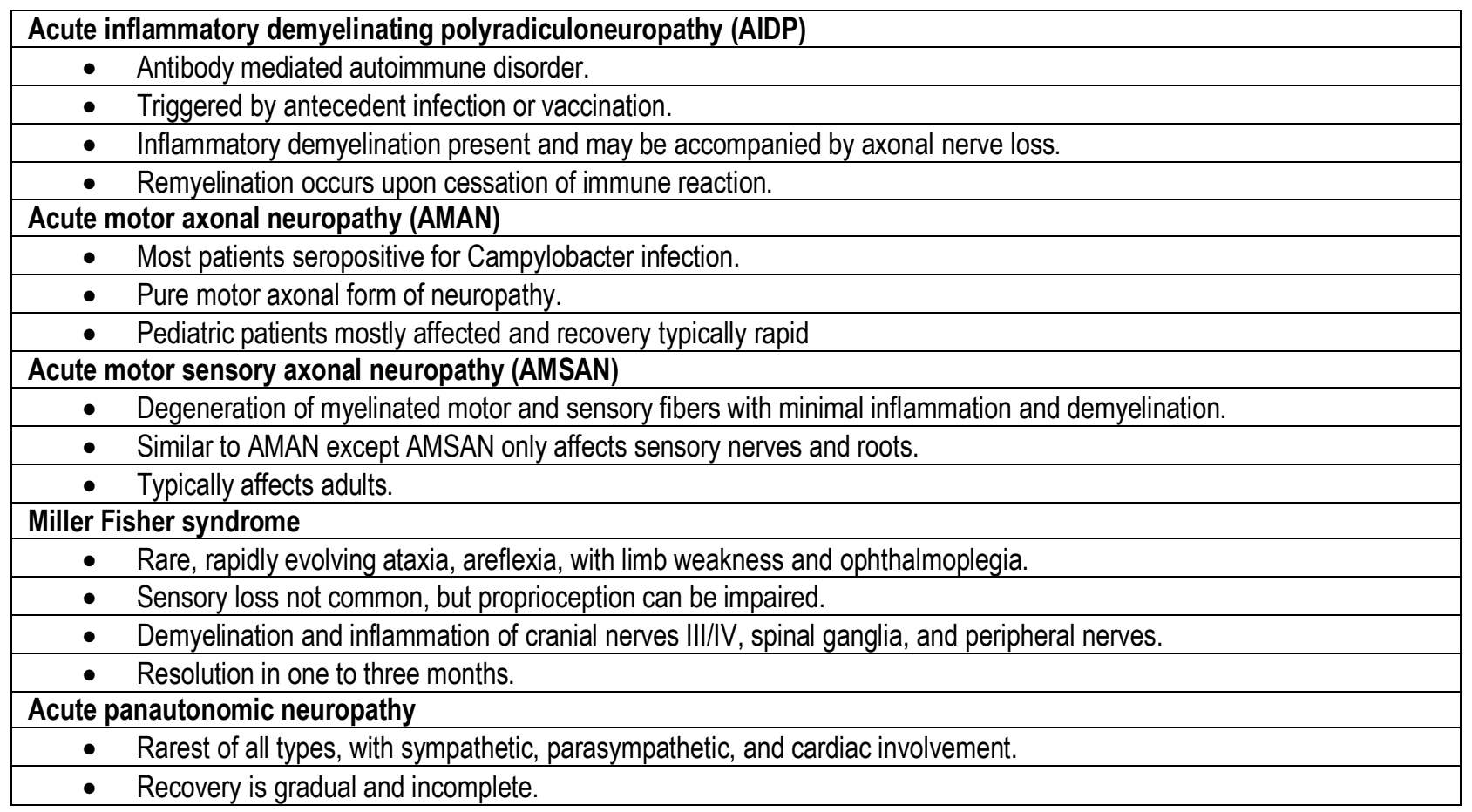

Note. From "Subtypes of Guillain-Barre' Syndrome," by Newswanger and Warren, 2004, American Family Physician, 69(10), p.4. Copyright 2004 by American Academy of Family Physicians. Adapted with permission.

Table 2: Clinical Features of Guillain-Barre' syndrome

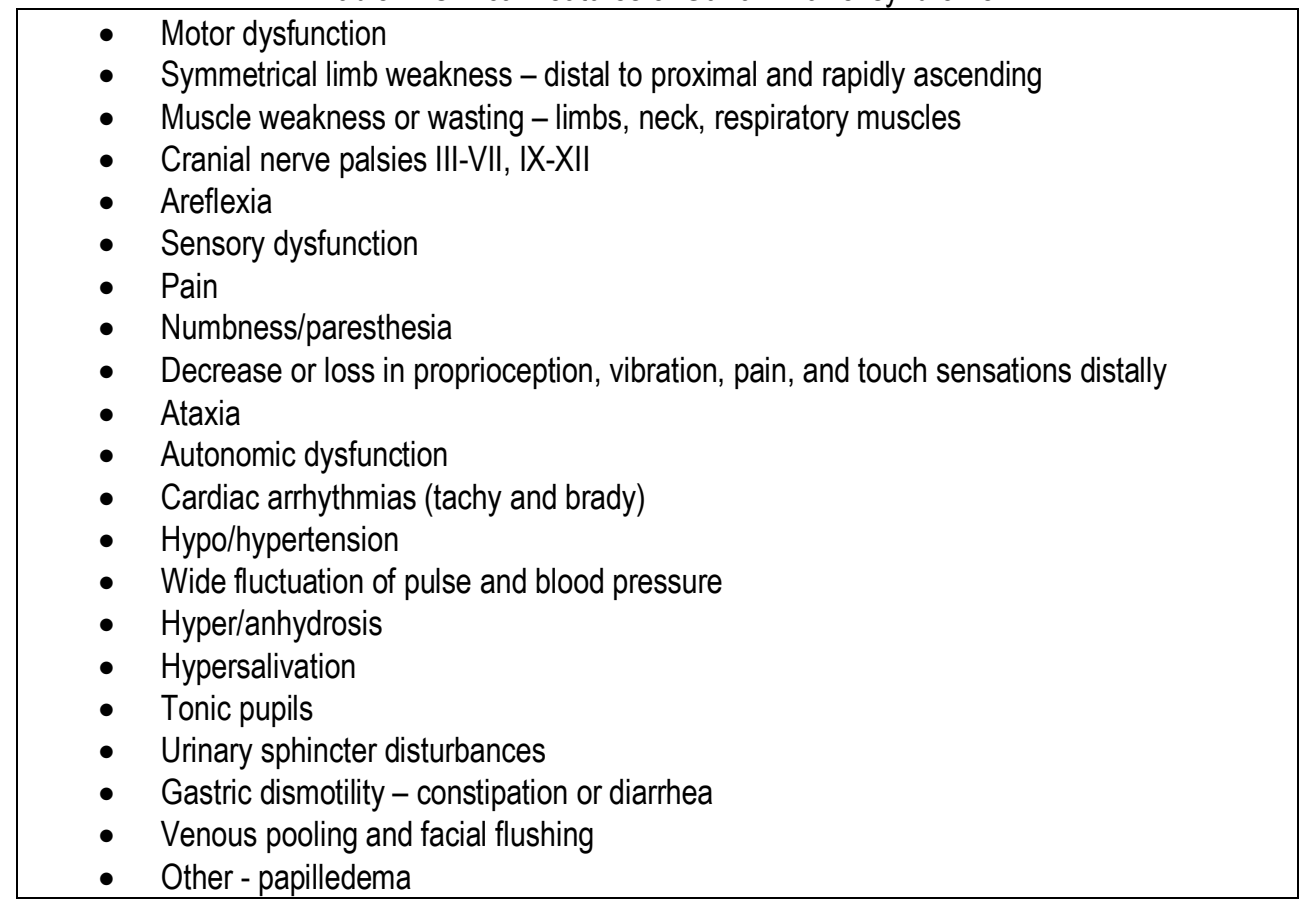


Many deaths due to GBS are attributed to autonomic disturbances; cardiac arrest being the most common cause, responsible for $20-30 \%$ of deaths. The most common symptoms are limb weakness and pain (see Table 2 for clinical signs and symptoms). Pain is experienced by approximately $90 \%$ of patients. ${ }^{2}$ The pain has been described as being like a "charlie horse," severe with slight movement that should not normally cause pain, as in hypersensitivity or hypalgesia. ${ }^{8}$

Although GBS is a peripheral neuropathy, there can be some cranial nerve involvement. The most common involvement is facial palsy. ${ }^{2}$ Diagnosis of GBS requires that the following criteria are met: progressive weakness of more than two limbs, areflexia, and progression of symptoms in four weeks or less. ${ }^{6}$ The neuropathy progresses and usually reaches a peak anywhere from 24 hours to four weeks with shorter or longer variations. The next phase is a plateau or stable phase that varies among patients, but usually lasts as long as the progressive stage. The recovery stage can be as long as one month to 2 years. Adults tend to show no improvement after two years. ${ }^{3}$ Complications or sequela of GBS can range from mild weakness to complete paralysis.

\section{Antecedent Events}

There are many factors strongly correlated with GBS diagnosis. According to Winer, many epidemiological studies corroborate that $75 \%$ of GBS patients document a preceding infection. ${ }^{6}$ Primary care providers (PCPs); are very likely to be consulted for the GBS patient's antecedent illness and should be prepared to identify early symptoms of GBS in the period following the antecedent illness. The emergency department (ED) also acts as primary care to many. Practitioners in the ED setting may be the first to see and examine patients with complaints that may manifest as GBS.

There are many antecedent events that may trigger GBS; however, only events commonly seen in primary care will be discussed. These events include bacterial infection, viral infections and vaccinations.

Bacterial Infection

The most frequent infection preceding GBS is due to the pathogen Campylobacter jejuni (C. jejuni). ${ }^{9}$ Bacteria from the Campylobacter genus are now identified as the most common source of bacterial gastroenteritis in the United States. Campylobacteriosis is an increasing problem. The rate of new Campylobacter cases currently exceeds that of the previously dominant salmonella and shigella. ${ }^{10}$

Bacterial gastroenteritis is found not only in underdeveloped countries, but also in many urban and industrialized societies. The Center for Disease Control (CDC) estimates that over 2.5 million cases of
Campylobacter enteritis occur each year in the United States alone. Food poisoning via $C$. jejuni is caused by consumption of raw or undercooked meat (mainly poultry), unpasteurized milk, home-canned foods, prepared foods (restaurants and home), or foods incubated in anaerobic environments. ${ }^{11}$

Contaminated water is also a source of infection mostly confined to developing, or poor and underdeveloped countries. Sequlae of $C$. jejuni infection not only includes GBS, but other morbidities such as reactive arthritis, pancreatitis, and carditis. ${ }^{12}$ The patient with gastroenteritis due to $C$. jejuni (if s/he seeks medical care) will most likely visit a primary care or acute care setting. These practitioners should be aware that this type of illness could trigger GBS. In studies done in the US, Europe, and Japan, cultures and serum have shown evidence of antecedent $C$. jejuni infection in $26-45 \%$ of GBS patients. These patients present with classic food poisoning type signs and symptoms, the most common being nausea, vomiting, abdominal pain, and diarrhea. Hadden and Gregson, ${ }^{9}$ reported that GBS preceded by a $C$. jejuni infection tends to have a more severe illness and worse prognosis when compared to other associated organisms or antecedent events. Poor long term prognosis can also be attributed to other factors including; old age, underlying disease, GBS disease severity (mechanical ventilation and rapid onset), and infection with $\mathrm{C}$. jejuni. It is speculated that immune response to an infection caused by $C$. jejuni causes increased axonal degeneration, which may regenerate, but often not completely. ${ }^{9}$

Research has been done to compare the presence of $C$. jejuni in peripheral blood mononuclear cells (PBMCs) of patients diagnosed with gastroenteritis due to $C$. jejuni and in healthy control subjects not exposed to the bacteria. The subjects studied consisted of 65 healthy persons, 10 patients diagnosed with $C$. jejuni induced enteritis, and 23 patients diagnosed with GBS. All GBS patients had preceding $C$. jejuni infection prior to GBS development. ${ }^{13}$ Tests were also done to determine the persistence of the Campylobacter organism. Testing was completed by obtaining a second blood sample approximately one year after the first sample was taken from the patients with known infection. Polymerase chain reaction (PCR) yielded identical results when follow-up samples were compared with the original samples. This data suggests that Campylobacter DNA is present and stable in the blood of a person for approximately one to two years.

This same study reported that human PBMCs contain Campylobacter DNA, and the highest percentages of Campylobacter DNA were found in the GBS patients with documented preceding $C$. jejuni infection. However, $C$. jejuni DNA was found in the healthy control group as well. One explanation for the presence of $C$. jejuni in the healthy

(c) The Internet Journal of Allied Health Sciences and Practice, 2007 
population is the constant presence of bacteria in foods consumed frequently, mainly poultry products. Other suggestions proposed by Van Rhijin's research team are that the bacteria are digested or die, but the DNA is not digested and remains within the host cells. This research does not prove that $C$. jejuni is the direct cause of GBS, but plays an important role in triggering the onset of GBS.

A second article examines a specific case in which an entire family suffered an outbreak of $C$. jejuni enteritis. Only one family member developed GBS during the outbreak. The importance of this data is to compare the immune response in the C. jejuni / GBS patient versus those only suffering from enteritis. This data may also aid in determining the factors that lead to GBS following a $C$. jejuni infection.

The patient that developed GBS was a ten year-old boy with preceding $C$. jejuni enteritis. The boy's father and brother also suffered from the same causal infection. Cultures were taken from all four of the family members, including the mother that was exposed but did not experience any enteritis. After immunologic testing, each family member was shown to have increased levels of antibodies IgA, IgM, and IgG. Dr. Ang and team, ${ }^{14}$ determined that the same microbe (C. jejuni) infected three individuals that were genetically related, and each person had a completely different immune response. This research determined there is no genetic link, and host-susceptibility factors determine what the immune response will be, which may or may not lead to the development of neurological symptoms such as GBS. ${ }^{14}$

Another bacterial trigger of GBS that is less prevalent is upper respiratory infection due to Mycoplasma pneumoniae found in about ten percent of GBS patients. ${ }^{2}$ This organism is difficult to isolate, therefore confirmation of preceding infection in GBS patients is difficult.

A case-control study was conducted to determine which antecedent infections are specifically associated with GBS. Participants examined were patients in the Dutch GuillainBarre' Trial. Jacobs et al., sampled 154 GBS patients and 154 sex and age matched controls with other neurological disease. 15 In the four weeks prior to GBS onset, 105 of 154 patients reported an antecedent illness. Multivariant analysis was performed and found that in the GBS population, serology was positive for the following antecedent infections: C. jejuni in $32 \%$ of patients, Cytomegalovirus (CMV) in 13\%, Epstein - Barr virus (EBV) in $10 \%$. In a univariant analysis, Mycoplasma pneumoniae was present in $5 \%$. All infections occurred significantly more often in GBS patients than in the control group. In the 49 patients not reporting antecedent illness, many had positive serology for antecedent infection. Serology showed C. jejuni in 29\%, CMV in 18\%, EBV in 16\%, and $\mathrm{M}$. pneumoniae in $4 \%$. It was also noted that 13 patients had positive serology for multiple infectious agents. It was concluded that $\mathrm{C}$. jejuni, CMV, EBV, and M. pneumoniae are specifically related to GBS, and are among the most common causes of antecedent infections. C. jejuni was concluded to be the most common bacterial antecedent infection and CMV the most common viral antecedent infection. 15

\section{Viral Infections}

The second most common antecedent infection reported is cytomegalovirus (CMV). CMV has been found in European studies to be present in GBS patients as an antecedent event in $11-22 \%$ of cases. ${ }^{2}$ According to the CDC, between $50 \%$ and $80 \%$ of adults in the US are infected with CMV by the age of 40 . CMV is largely a silent virus therefore; actual incident rates are not available. GBS with preceding CMV infection tends to be more prevalent in young females, leading to a more complicated disease process with severe respiratory complications and liver disease. ${ }^{16}$

Acute acquired CMV is similar to mononucleosis in presentation and is characterized by fever, malaise, myalgia, arthralgia, splenomegaly, and cervical lymphadenopathy. ${ }^{17}$ These complaints are again reasons for the patient to visit a PCP. The practitioner should consider GBS when a patient has a previous diagnosis of CMV in their past medical history. It is postulated that GBS is more prevalent in the young adult population because of its similarity to mononucleosis. Mononucleosis, like CMV, is most common in teens and young adults. Numazaki \& Chiba, ${ }^{18}$ reported that anywhere from $40-100 \%$ of the general population are infected with, or have latent CMV infections.

Patients with a preceding diagnosis of CMV will have a different course of GBS as opposed to the patient with the C. jejuni infection. The CMV patient will tend to develop cranial nerve palsies bilaterally, have severe respiratory complications (e.g.; mechanical ventilation), and experience severe sensory losses.

There are many other viruses that have been suggested to incite GBS, but have not been extensively studied. Epstein - Barr virus (EBV) is prevalent in about $10 \%$ of GBS cases. ${ }^{3}$ HIV associated GBS has been present in medical literature since the mid 1980's. HIV preceded GBS appears in the early and late stages of HIV, before and during seroconversion. Many HIV patients have a co-diagnosis of CMV. Studies have not discerned whether HIV or CMV is the trigger in these particular patients. GBS triggered by HIV tends to be associated with recurrent episodes of GBS, and is more likely to be classified as chronic inflammatory demyelinating polyradiculoneuropathy (CIDP). ${ }^{19}$ 
Other viral infections known to trigger GBS less frequently are Herpes Simplex 1 and $2, H$. influenzae, influenza $A$ and $B$ viruses, varicella-zoster viruses, hepatitis $A, B, C$, and $D$.

\section{Vaccinations}

There is controversy surrounding whether or not vaccinations can trigger GBS because of mass vaccination of children and adults worldwide. Vaccinations are essentially a requirement for all children entering school and for most students entering college. The most common and recent vaccine implicated in triggering GBS is the influenza vaccine. One study examined the 1992-1993 and 1993-1994 influenza seasons and GBS cases reported during that time, and found the adjusted relative risk of 1.7 cases per 1 million influenza vaccinations. The risk of the flu vaccine inciting a case of GBS was one to two cases per $1,000,000$ people vaccinated. ${ }^{8}$

The CDC has established a reporting system known as the Vaccine Adverse Event Reporting System (VAERS) ${ }^{20}$ that stated in its surveillance study in 2003, "The risk of developing vaccine-associated GBS is less than the risk of severe influenza." ${ }^{\prime}$ The numbers of individual case reports of vaccine related GBS events were prevalent enough to warrant attention and further studies. Nationwide surveillance began as a result of the New Jersey "swine flu" vaccination program in 1976-1977. In 1976 the CDC initiated a massive immunization program to avoid an influenza pandemic similar to the "killer flu of 1918" because one soldier died earlier in the year (1976) of a similar antigenic strain of flu as that of 1918. The mass vaccination ceased in December of 1976 because the threat of an epidemic decreased dramatically. Roughly 43 million people were vaccinated during this time frame of October-December 1976. The CDC initiated studies regarding the relative risk of this vaccine triggering GBS and the relative risk was shown to be 7.1. The swine flu vaccination was attributed to eight to nine cases of GBS per million vaccinations. This study also stated that the attributable risk of developing GBS after receiving the flu vaccine is one to two cases per million vaccinated. Several studies have determined a clear association between the swine flu vaccine and GBS if the onset is within a set amount of weeks after vaccination; however a clear causal association has yet to be established. ${ }^{21}$

There are many documented cases of influenza vaccines being the antecedent event triggering GBS, but studies have yet to show that the risks outweigh the benefits of receiving the influenza vaccine. The VAERS of the CDC compiled reports from 1990 to 2003 regarding the incidence of influenza vaccine and GBS diagnosis. The study concluded that reporting rates of GBS after influenza vaccination have decreased; however, long onset interval and the lowered possibility of other preexisting illnesses contributing to GBS, the findings state the possibility of a causal relationship between GBS and the influenza vaccine. ${ }^{22}$

Over 61 million flu vaccines were given during the 20042005 flu season and over 81 million doses have been given currently for the 2005-2006 flu season. ${ }^{20}$ Although epidemiological studies state that the influenza vaccine is safe and the risk of GBS is minimal, the risk is still present and patients should be educated about the possible risk involved regarding GBS and vaccination.

Other vaccines have been implicated as triggers for GBS, but the cases are considered anecdotal. Risk has yet to be established due to the small number of cases to be studied. The vaccines implicated are the polio, rabies, tetanus toxoid, MMR, DTP, hepatitis $B$, hepatitis $A$, and meningitis. Patients with GBS like symptoms should be asked about any recent vaccines. Post vaccination symptoms of paralysis or hyporeflexia should lead primary care providers to consider GBS in their differential.

\section{Summary}

The PCP is likely to be the port of entry when a patient has complications due to a viral-bacterial infection or a vaccination. GBS complications can take hours to weeks to manifest and in severe cases may require mechanical ventilation. Twenty-five percent of all GBS patients require ventilator support of some type. ${ }^{2}$ PCP's must recognize antecedent events, signs, and symptoms of GBS to facilitate treatment immediately. Education will also prevent iatrogenic relapses in patients with a history of GBS. Approximately $25 \%$ of deaths occur within the first week of onset of GBS. ${ }^{2}$ Initiating supportive care and therapeutic treatment can decrease the amount of recovery time and amount of residual debilitating effects of GBS. Twenty to thirty percent of GBS patients are left with some form of residual weakness or disability. ${ }^{6}$

GBS is rare and practitioners may not recognize GBS when it does present. Therefore, early recognition of antecedent events, signs and symptoms, and knowledge of GBS, with attention to potential outcomes, will decrease mortality and residual effects of GBS. Although GBS is a rare syndrome it is not rare to the person that has it.

\section{Results}

GBS is a rare autoimmune syndrome. Bimodal incidence is found to be higher in the young adult and elderly populations. A case study examined an outbreak of gastroenteritis within a family. All family members were exposed to $C$. jejuni, but only one person developed GBS. This proves no genetic link. Many antecedent events are strongly correlated with a GBS diagnosis. The exact cause of GBS is unknown, but many triggers have been well established. Onset of GBS occurs one to three weeks after a preceding illness, and $75 \%$ of GBS patients document a

(c) The Internet Journal of Allied Health Sciences and Practice, 2007 
preceding illness. The most common infection preceding GBS is C. jejuni gastroenteritis. ${ }^{7}$ Research with $C$. jejuni DNA has not proven that $C$. jejuni infection is a direct cause of GBS. However, evidence shows $C$. jejuni and M. pneumoniae to be specifically related to GBS. ${ }^{15}$

The second most common antecedent infection preceding GBS is CMV. CMV is present in most adults as a latent infection. Studies show an increased prevalence of CMV triggered GBS in young female populations. Research has shown that a preceding CMV infection can lead to a more severe and complicated disease process with increased residual complications because of increased cranial nerve involvement and increased sensory and motor loss. Evidence shows CMV and EBV to be specifically related to GBS as well. ${ }^{15}$ Other viruses known to trigger GBS include HIV, influenza, herpes, and numerous others. Of these other viruses thought to be triggers, little information is available to study due to low incident rate.

Another class of GBS triggers under extensive investigation is vaccinations. Extensive past and present research regarding the relationship between vaccines and GBS exists. Recent research has focused on the influenza vaccine. The outcome of most studies state that the risk of acquiring GBS is minimal. The CDC's Vaccine Adverse Event Reporting System (VAERS) for 2003 states, "The risk of developing vaccine-associated GBS is less than the risk of severe influenza". ${ }^{8}$ Several studies have determined a correlation between the influenza vaccine and GBS, but a clear causal association has not been established. Studies cannot prove that risk outweighs the benefit regarding vaccinations versus increased risk of GBS.

\section{Discussion}

Many studies have proven that antecedent infection and/or events trigger GBS, but do not directly cause GBS. Practitioners are also able to predict any possible complications of the disease and any possible residual effects based on past research. Many bacterial and viral triggers have been studied, but not as extensively as $C$. jejuni and CMV. Triggers that have not been studied extensively may be as important to understanding onset of GBS. Lesser-studied viruses include herpes, EBV, HIV, influenza, varicella, and hepatitis. Much more research is needed regarding these antecedent infections, but lack of case studies makes this research difficult. A PCP will most likely address many of the antecedent bacterial-viral infections and/or post-illness complications. They should be suspicious for any arising post-illness complications resembling any GBS symptoms. "The diagnosis of GuillainBarre' Syndrome itself is usually not difficult for the neurologist, but can be challenging for the doctor of first contact who may not have seen a case since medical school". ${ }^{5}$ These antecedent illnesses are prevalent in society. PCP's understanding what agents are associated with GBS benefits the patient by enabling early diagnosis and treatment resulting in decreased morbidity and mortality. PCPs can also educate patients about the risk of GBS with vaccination/illness, what signs and symptoms to look for, and when to return to the clinic if GBS is suspected. The patient presenting with post-febrile weakness may be dismissed and sent home. In this case it is critical for the PCP to know that even though differential diagnosis is broad, it is critically dependent upon them first recognizing the onset of GBS or ruling it out.

\section{Conclusion}

The prognosis of GBS is dependent upon early diagnosis and intervention. Early diagnosis begins with the PCP, therefore the PCP must recognize patients at risk and consider GBS in the differential diagnosis. Antecedent events encountered in primary care include, but are not limited to, gastroenteritis, upper respiratory infection, cytomegalovirus, Epstein-Barr virus, and vaccinations against influenza, meningitis, and tetanus toxoid. Although GBS is rare, further research of triggers is warranted. Influenza vaccines are traditionally made in chicken eggs where Campylobacter is endemic; consequently research is necessary in this field because of mass immunization programs that are especially vigilant in high risk/vulnerable populations. If direct causes could be identified, prophylaxis and treatment options (other than supportive care) could be developed. No genetic link has been established, but no explanation exists regarding why one family member has GBS and the others do not. More research is needed to determine why the other family members did not get GBS. Much is still unknown about GBS (cause, pathophysiology, treatment, and recovery/prognosis) therefore, future research has endless limits. PCPs are the "gatekeepers" to healthcare. They must know the common illnesses and vaccines shown to trigger GBS, and must recognize signs and symptoms of GBS when present in the acute care setting. Education of providers is the key to decreasing mortality and sequela through PCP's early recognition and intervention.

\section{References}

1. Nachamkin, I., Allos, B. M., \& Ho, T. (1998, July). Campylobacter Species and Guillain-Barre' Syndrome. Clinical Microbiology Reviews, 11, 3. Retrieved November 19, 2005, from http://www.ncbi.nlm.nih.gov/entrez

2. Seneviratne, U. (2000, April 19). Guillain-Barre' syndrome. Institute of Neurology, April, 2000. Retrieved November 20, 2005, from http://www.ncbi.nlm.nih.gov

(c) The Internet Journal of Allied Health Sciences and Practice, 2007 
3. Li, M. (2005, July 5). Guillain-Barre syndrome Basics. Retrieved January 25, 2006, from http://online.statref.com

4. Joseph, S. A., \& Tsao, C. (2002, October). Guillain-Barre' syndrome. Adolescent Medicine, 13. Retrieved January 27, 2006, from http://home.mdconsult.com/das/article

5. Hughes, R. A., \& Cornblath, D. R. (2005, November 5). Guillain-Barre' syndrome. Retrieved January 6, 2006, from www.thelancet.com

6. Winer, J. B. (2001, March 8). Guillain Barre' syndrome. J Clin Pathol: Mol Pathol, 54, 381-385. Retrieved November 22, 2005, from www.molpath.com

7. Hadden, R. D., \& Gregson, N. A. (2001). Guillain-Barre' syndrome and Campylobacter jejuni infection. Journal of Applied Microbiology, 90, 145S-154S.

8. Newswanger, D. L., \& Warren, C. R. (2004, May 15). Guillain-Barre' Syndrome. American Family Physician, 69, 10. Retrieved January 25, 2006, from http://mdconsult.com/das/article/body

9. Hadden, R., Gregson, N., Gold, R., Wilson, H., \& Hughes, R. (2001). Guillain-Barre' syndrome serum and antiCampylobacter antibody do not exacerbate experimental autoimmune neuritis . Journal of Neuroimmunology, 119(2), 306-316.

10. Friedman, C., Hoekstra, R., Samuel, M., Marcus, R., Bender, J., \& Reddy, S. et al. (2004). Risk Factors for Campylobacter infection in the United States: A Case-Control Study in FoodNet Sites. Clinical Infectious Diseases, 38, S285-S296. Retrieved April 4, 2006, from http://www.cdc.gov/foodnet/pub/cid

11. Bradford, J. (2005, July 5). Botulism. Retrieved January 25, 2006, from http://online.statref.com

12. Mishu, B., llyas, A. A., Koski, C. L., Vriesendorp, F., Cook, S. D., \& Mithen, F. A. et al. (1993, June 15). Serologic Evidence of Previous Campylobacter jejuni infection in patients with the Guillain-Barre Syndrome. Annals of Internal Medicine, 118, 12, 947-953. Retrieved January 29, 2006, from http://www.annals.org

13. Van Rhijin, Bleumink-Pluym, N., Van Putten, J., \& Van den Berg, L. (2002). Campylobacter DNA is present in circulating myelomonocytic cells of healthy persons and in persons with Guillain-Barre' Syndrome. Journal of Infectious Diseases, 185(2), 262-268.

14. Ang, C., Van Doorn, P., Enoltz, H., Merkies, I., Jacobs, B., \& Van de Klerk, M. et al. (2000). A case of Guillain-Barre' syndrome following a family outbreak of Campylobacter jejuni enteritis. Journal of Neuroimmunology, 111, 229-233.

15. Jacobs, B., Rothbarth, P., Van der Meche, F., Herbrink, P., Schmitz, P., \& DeKlerk, M. et al. (1998, October 1). The spectrum of antecedent infections in Guillain-Barre' Syndrome: a case-control study. Neurology, 51(4), 1110-5. Retrieved April 4, 2006, from http://home.mdconsult.com.chekov.evms.edu

16. Visser, L. H., Van der Meche, F. G., Meulstee, J., Rothbarth, P., Jacobs, B. C., \& Schmitz, P. I. et al. (1996, September). Cytomegalovirus infection and Guillain-Barre syndrome: The clinical, electrophysiologic, and prognostic features. American Academy of Neurology, 47(3), 668-673. Retrieved January 22, 2006, from http://gateway.ut.ovid.com

17. Tierney, L. M., Jr., Mcphee, S. J., \& Papadakis, M. A. (2005). Current Medical Diagnosis \& Treatment (44th ed.). New York: McGraw Hill.

18. Numazaki, K., \& Chiba, S. (1997, November). Cytomegalovirus infection and Guillain-Barre' syndrome. American Academy of Neurology, 49(5), 1472-1473. Retrieved January 27, 2006, from http://gateway.ut.ovid.com

19. Brannagan, T. H., \& Zhou, Y. (2003, April 15). HIV-associated Guillain-Barre syndrome. Retrieved January 25, 2006, from http://www.ncbi.nlm.nih.gov/entrez

20. Center for Disease Control (2006, January 23). Vaccine Supply and Prioritization Recommendations for the U.S. 200506 Influenza Season. Retrieved January 28, 2006, from http://www.cdc.gov/flu

21. Freedman, D. A., \& Stark, P. B. (1999, August 15). The Swine Flu Vaccine and Guillain-Barre' Syndrome: A case study in relative risk and specific causation. Retrieved November 20, 2005, from http://www.pubmed.com

22. Haber, P., DeStefano, F., Angelo, F. J., Iskander, J., Shadomy, S. V., \& Weintraub, E. et al. (2004, November 24). Guillain-Barre Syndrome Following Influenza Vaccination. JAMA, 292(20), 2478-2481. Retrieved January 27, 2006, from www.jama.com 